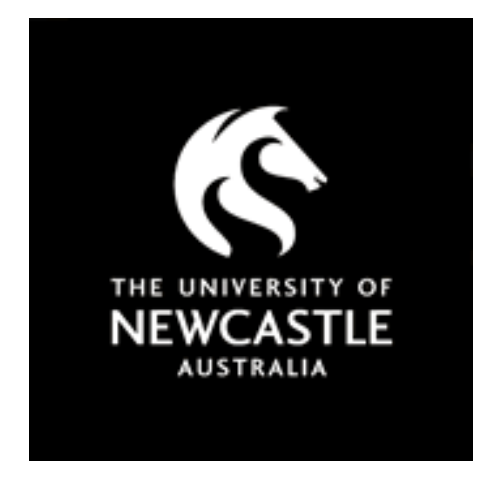

NOVA

University of Newcastle Research Online

nova.newcastle.edu.au

Smith, Jacob; Webber, Grant Bruce; Warr, Gregory G.; Atkin, Rob. "Silica particle stability and settling in protic ionic liquids". Originally published in Langmuir Vol. 30, Issue 6, p. 1506-1513 (2014)

Available from: $\underline{\text { http://dx.doi.org/10.1021/la403978b }}$

This document is the Accepted Manuscript version of a Published Work that appeared in final form in Langmuir, copyright (C) American Chemical Society after peer review and technical editing by the publisher. To access the final edited and published work see http://dx.doi.org/10.1021/la403978b

Accessed from: http://hdl.handle.net/1959.13/1041241 


\section{Silica Particle Stability and Settling in Protic Ionic}

\section{Liquids}

Jacob Smith, ${ }^{\ddagger}$ Grant Bruce Webber, ${ }^{\ddagger}$ Gregory G. Warr ${ }^{\S}$ and Rob Atkin *¥

${ }^{\ddagger}$ Priority Research Centre for Advanced Particle Processing and Transport

The University of Newcastle, Callaghan, NSW 2308, Australia

$\S$ School of Chemistry, The University of Sydney, NSW 2006, Australia

*To whom correspondence should be addressed.

\section{Abstract}

$10 \mathrm{wt} \%$ silica particle suspensions have been investigated in the protic ionic liquids (ILs) ethylammonium nitrate (EAN), ethanolammonium nitrate (EtAN), propylammonium nitrate (PAN) and dimethylethylammonium formate (DMEAF). Static and dynamic light scattering reveal that single particles co-exist in dynamic equilibrium with flocculated networks at room temperature. These types of systems are classified as weakly flocculated, and are quite rare. As weakly flocculated systems generally exist only within a narrow range of conditions, the effect of temperature was probed. When temperature is increased the thermal motion of suspended particles increases, favouring dispersion, but in ILs suspensions, heating reduces the stabilising effect of the interfacial structure of the IL. When subjected to a small increase in temperature, particle suspensions in ILs become unstable, indicated by the absence of a peak corresponding to single particles in the light scattering data. For EAN and DMEAF, further increasing temperatures above $40{ }^{\circ} \mathrm{C}$ returns the systems to a weakly flocculated state in which thermal energy is sufficient to break particles away from aggregates. Weakly flocculated suspensions in EAN and EtAN settle more rapidly than predicted by the Stokes equation, as the particles spend a significant portion of time in large, rapidly-settling flocs. Surprisingly, suspensions in PAN and DMEAF settle slower than predicted. Oscillatory rheology indicates that these suspensions are viscoelastic, due to a 
persistent, long-range structure in the suspension that slows settling. In aggregated systems settling is very rapid.

\section{Introduction}

Ionic liquids (ILs) are solvents composed entirely of ions. ILs can be subcategorised in several, non-exclusive ways, including as room temperature ILs which have melting points below $25^{\circ} \mathrm{C}$; as protic ILs, which are formed by Brønsted-Lowry proton transfer; and as aprotic ILs, which are formed by other synthetic pathways, including charge transfer metathesis reactions for the popular aprotic class. ${ }^{1-9}$ ILs often possess potentially useful properties including low vapour pressure, high thermal and electrochemical stability, and high conductivity, amongst others, and are attracting research interest in a diverse range of fields. ${ }^{10-29}$ IL-based nanofluids, such as the suspensions investigated herein, have been studied for particle synthesis and catalysis, ${ }^{1,17,30-33}$ heat transfer, ${ }^{23,34-}$ ${ }^{36}$ in green chemical applications ${ }^{18}$ and magnetofluids. ${ }^{20-22,37}$

Considerable progress has been made elucidating the bulk nanostructure of ILs over the past few years. ${ }^{7,19,38-43}$ Scattering experiments ${ }^{41,42,44-47}$ have shown that protic ILs are often nanostructured in the bulk liquid. Nanostructure results from electrostatic attractions (and often hydrogen bonding ${ }^{46}$ ) between charged groups, which leads to the formation of polar domains. Cation alkyl chains are expelled from these charged regions via a solvophobic effect ${ }^{48}$ and cluster together to form apolar domains within the liquid. ethylammonium nitrate (EAN) and propylammonium nitrate (PAN) both have sponge-like bulk nanostructure. ${ }^{41,42}$ Segregation between polar and apolar domains is more distinct in PAN due to the longer cation alkyl chain producing a stronger solvophobic effect. ${ }^{41}$ Ethanolammonium nitrate (EtAN) has a different, clustered bulk nanostructure, ${ }^{42}$ because the alcohol group attached to the cation chain disrupts solvophobic interactions, and can hydrogen bond with the charge centres. The effect of adding alkyl chains to the cationic group on liquid nanostructure has been probed using dimethylethylammonium formate (DMEAF) because substituted ammonium nitrates are frequently explosive. The bulkiness and 
limited H-bonding capacity of the cation prevents the formation of well-defined regions within the liquid, and less segregated bulk structure results. ${ }^{44}$

Bulk IL nanostructure becomes more ordered at macroscopic solid ${ }^{49}$ and air interfaces. ${ }^{50}$ For liquids that have sponge-like nanostructure, like EAN and PAN, the bulk morphology is transformed into a more ordered layered structure at the surface, reminiscent of surface-induced sponge-to-lamellar transitions that occur in lyotropic systems. ${ }^{51}$ For less structured liquids like EtAN and DMEAF, layers of ions similar to molecular liquid solvation layers form. There are five main factors that control the level of interfacial order in pure ILs: the degree of bulk structure ${ }^{24,42}$ the strength of interaction between the surface and the cation, which can be electrostatic ${ }^{49}$ or solvophobic; the molecular flexibility of the ions; ${ }^{49}$ the interfacial roughness; ${ }^{52}$ and the temperature. ${ }^{49}$ Small amounts of dissolved solutes such as water or inorganic salts have been shown to reduce interfacial structure near solid surfaces. ${ }^{53,54}$ For negatively charged surfaces, such as silica, the IL cation's charged group is attracted to the surface to neutralise the charge. ${ }^{55}$ Attached apolar groups are projected away from the surface into the bulk, which attract a further layer of cations solvophobically by their alkyl tails. The charged groups of those cations attract anions, sufficient to neutralise the charge, which attract more anions by pi-pi stacking, which attract a new layer of cation charged groups. This sequence continues with ever reducing fidelity until the structure decays to that of the bulk. ${ }^{27,55-57}$

In a previous article the stability and settling of $10 \mathrm{wt} \% 1 \mu \mathrm{m}$ silica particles in pure water, pure EAN and mixtures of various compositions of these two miscible solvents was reported. ${ }^{54}$ Dynamic light scattering (DLS) measurements revealed a single peak at $1 \mu \mathrm{m}$ for pure water and pure EAN suggesting that these suspensions were stable to aggregation, but that larger objects - aggregates were present in all EAN/water mixtures. Stability in pure water is expected, due to silica's high negative charge at ambient $\mathrm{pH}$. Particle instability in the mixed solvents was thought to be a 
consequence of EAN raising the ionic strength and thereby screening silica's surface charge such that attractive van der Waals forces between particles dominated. Particle stability in pure EAN was surprising, given that silica's native surface charge will be completely screened by the $11 \mathrm{M}$ ionic strength of EAN. This stability was interpreted to be a consequence of EAN's known interfacial layering producing structural forces that were sufficiently strong to prevent particle aggregation. In a manner analogous to steric stabilisation of particles by adsorbed polymer, these structural forces prevent particle contact and thus stabilise the suspension. Similar results have been reported for particles in aprotic imidazolium ILs. ${ }^{25}$ Even though the silica particles in EAN appeared to be stable to aggregation, they inexplicably settled $\sim 6$ times faster than predicted by a modified form of the Stokes equation. In this work $10 \mathrm{wt} \% 1 \mu \mathrm{m}$ silica particle suspensions in EAN, PAN, EtAN and DMEAF are examined using DLS, static light scattering (SLS) and oscillatory rheology, and settling rates are compared to those predicted by the Stokes equation. These experiments elucidate the origin of the single particle peak in DLS spectra and faster than expected settling.

The modified Stokes equation accurately predicts the settling velocity $(\omega)$ of multiple spheres settling in a liquid:

$$
\omega=\frac{\left|\rho_{p}-\rho_{f}\right| g r^{2}}{18 \mu} \times \frac{[1-\phi]}{\left[1+\frac{4.6 \phi}{1-\phi}\right]}
$$

The left half of the equation is the classical Stokes model, where; $\rho_{p}$ and $\rho_{f}$ are the densities of the particle and fluid respectively, $r$ is the particle radius, $\mu$ is the dynamic viscosity. The multiplier on the right is a modification employed to account for the effects of particle interactions on settling velocity at higher particle volume fractions, where $\phi$ is particle volume fraction. As settling velocity is proportional to the square of the particle radius, when particles aggregate, observed settling rates are faster than predicted by the Stokes equation. 


\section{Experimental Section}

Nitrate ILs were prepared by slow addition of nitric acid (Merck) to ethylamine, propylamine, or ethanolamine (Aldrich or Fluka). Equimolar amounts were mixed in an aqueous solution below $10^{\circ} \mathrm{C}$. Excess water was removed by rotary evaporation followed by nitrogen purging and heating at $105-110^{\circ} \mathrm{C}$ overnight. DMEAF was prepared by slow addition of equimolar formic acid (Fluka) to dimethylethylamine (Aldrich). Excess water was removed by rotary evaporation at temperatures below $30^{\circ} \mathrm{C}$. The water content of nitrate ILs was undetectable Karl-Fischer titration, while the water content of DMEAF was approximately $0.1 \mathrm{wt} \%$. Silica particles $(1 \mu \mathrm{m}$, dry from the Fiber Optics Centre, California) were dried overnight in a vacuum oven at $\sim 40^{\circ} \mathrm{C}$ and $10-40 \mathrm{mBar}$ immediately before use.

IL densities were determined using an Anton-Paar DMA 5000 M density meter. Silica particle density was measured using a pycnometer. IL refractive indices were measured as a function of temperature using a benchtop refractometer with integrated water collar and thermostat. The refractive index of silica can be determined from the measured density. ${ }^{58}$

Dispersion stability was assessed by static light scattering (SLS) using a Malvern Instruments Mastersizer 2000, which can measure particle sizes up to $1 \mathrm{~mm}$. IL particle dispersions were ultrasonicated in a water bath for at least 15 minutes immediately prior to measurement. A small volume cell $(\sim 20 \mathrm{~mL})$ was used. Background scattering profiles for the pure IL were taken, and then the particle dispersion particle size distributions were measured. Measurements were taken every 5 minutes for an hour, then every 15 minutes for an extra 2 hours following. Experiments were ended after 2 hours because water ingression into the sample became unacceptably high ( $0.25 \mathrm{wt} \%)$.

Temperature-dependent light scattering measurements could not be performed on the Mastersizer 2000, so a Malvern Instruments NanoSeries ZetaSizer Nano-ZS dynamic light scatterer (DLS) was used. Samples were sonicated to ensure dispersion, placed in a stoppered, sealed glass 
cuvette, and inserted into the instrument. The temperature of the instrument was then rapidly equilibrated to the desired value and measurements commenced. Measurements of particle size distribution were recorded every 10 minutes for at least 24 hours. Water content was assessed after the experiment and was invariant: undetectable by Karl-Fisher titration for the nitrate ILs, and less than 0.25 wt $\%$ for DMEAF.

For settling experiments, particle suspensions in the ILs were prepared by weighing the dry particles into a $3.5 \mathrm{~mL}$ sample tube then adding IL to produce a $10 \mathrm{wt} \%$ dispersion. A $10 \mathrm{wt} \%$ dispersion of silica particles in Milli-Q water was used as a control; the settling rate for this suspension followed that predicted by the Stokes equation. Water ingression into IL samples was prevented using screw top lids sealed using Teflon tape and lab parafilm. The particles were then dispersed in the liquid by ultrasonication. Before each experiment all samples were placed in an ultrasonic bath for 20 minutes and inverted three times to disperse the particles. Settling experiments were performed in a backlit, glass walled, hot water bath to facilitate temperature control. The temperature of the water bath was stable to $\pm 0.5^{\circ} \mathrm{C}$. Photographs were taken at regular time intervals using a digital camera and the distance between the settling front and the meniscus of the suspension was determined for each photograph. The distance of the settled front from the meniscus was plotted as a function of time, yielding the velocity of the settling front. Following experiments, selected samples were re-analysed by Karl-Fischer titration. In every case the water content was unchanged.

Rheological measurements were performed using a TA Instruments AR-G2 rheometer with a cone and plate. The cone of radius was $40 \mathrm{~mm}$ and the angle was $1^{\circ} 59^{\prime} 36^{\prime \prime}$. A portion of each sample was retained after measurement and analysed by Karl-Fischer titration. The water content increased by no more than $0.1 \%$ w/w for any sample. Temperature was controlled using a Peltier plate and jacket with chilled water. Rheological data was measured five times each on samples from three separate IL sample batches (for each IL) and averaged. The large number of repetitions were 
performed to ensure there were no differences in rheological properties of the ILs dependent on synthetic batch. The variance between rheological data measured was less than $5 \%$ for all experiments. Temperature dependent viscosity was measured at a constant shear rate that fell within the Newtonian response range for each liquid. The temperature was increased from $20^{\circ} \mathrm{C}$ to $50^{\circ} \mathrm{C}$ over a 30 minute period and the viscosity measured at each degree. Shear dependent viscosities were measured by sweeping the shear rate from $1 \mathrm{~s}^{-1}$ to $4000 \mathrm{~s}^{-1}$. Each data point represents an average of 5 consecutive measurements that were within $5 \%$ of one another at each shear rate.

\section{Results and Discussion}

Physical properties required for determining particle stability by light scattering (density ( $\rho$, g.cm ${ }^{-3}$ ) and refractive index (RI)) and to predict particle settling rates using the Stokes equation (liquid density and viscosity $(\mu, \mathrm{cP})$ ) are presented in Table 1 as a function of temperature for EAN, PAN, EtAN and DMEAF. The lifetime of the single particle peak from DLS experiments, suspension settling rates $\left(\boldsymbol{\omega}_{\text {obs }}\right)$ and the ratio of the measured settling rate to Stokes predictions $\left(\frac{\omega_{\text {obs }}}{\boldsymbol{\omega}_{\text {calc }}}\right)$ are also shown.

Static light scattering particle size distribution data measured at $20^{\circ} \mathrm{C}$ for $10 \mathrm{wt} \% 1 \mu \mathrm{m}$ silica particles dispersed in EAN, EtAN, PAN and DMEAF 30 minutes after ultrasonication are presented in Figure 1. For all the ILs, measurements immediately after ultrasonication revealed only a single peak at $1 \mu \mathrm{m}$, indicating that the particles were effectively dispersed. The particle size distribution of silica particles in water exhibits a single peak at $\sim 1 \mu \mathrm{m}$ indefinitely. 
Table 1. The density $\left(\rho, \mathrm{g} . \mathrm{cm}^{-3}\right)$, refractive index (RI), dynamic viscosity $(\mu, \mathrm{cP})$, lifetime of single particle peak (hours before the single particle peak begins to be lost or other aggregate peaks form), observed settling rate $\left(\boldsymbol{\omega}_{\boldsymbol{o b s}}, \times 10^{-8} \mathrm{~m} . \mathrm{s}^{-1}\right)$, and ratio of observed to predicted settling rates $\left(\boldsymbol{\omega}_{\text {obs }}: \boldsymbol{\omega}_{\text {calc }}\right)$ for $10 \mathrm{wt} \%$ dispersions of $1 \mu \mathrm{m}$ silica spheres in the ionic liquids studied, at various temperatures $\left(\mathrm{T},{ }^{\circ} \mathrm{C}\right)$.

\begin{tabular}{|c|c|c|c|c|c|c|c|c|c|c|c|c|}
\hline \multirow[b]{2}{*}{$\mathrm{T}$} & \multicolumn{6}{|c|}{ EAN } & \multicolumn{6}{|c|}{ EtAN } \\
\hline & $\mathrm{P}$ & RI & $\mu$ & $\begin{array}{c}\text { DLS Single } \\
\text { particle peak } \\
\text { lifetime }(\mathrm{h})\end{array}$ & $\omega_{o b s}$ & $\frac{\omega_{o b s}}{\omega_{c a l c}}$ & $\rho$ & RI & $\mu$ & $\begin{array}{l}\text { DLS Single } \\
\text { particle peak } \\
\text { lifetime (h) }\end{array}$ & $\omega_{o b s}$ & $\frac{\omega_{\text {obs }}}{\omega_{\text {calc }}}$ \\
\hline 20 & 1.214 & 1.4535 & 36 & $>6$ months & 7.20 & 6.73 & 1.385 & 1.4850 & 157 & $>6$ months & 0.293 & 1.52 \\
\hline 25 & 1.210 & & 32 & $>24$ & & & 1.382 & & 123 & & & \\
\hline 30 & 1.207 & 1.4515 & 29 & 5 & 24.7 & 18.44 & 1.379 & 1.4840 & 93 & $>24$ & 0.478 & 1.46 \\
\hline 35 & 1.204 & & 25 & 3 & 36.1 & 23.59 & 1.376 & & 69 & & & \\
\hline 40 & 1.201 & 1.4505 & 22 & $>24$ & 33.0 & 18.54 & 1.373 & 1.4835 & 49 & & & \\
\hline 50 & 1.195 & 1.4475 & 15 & $>24$ & 20.5 & 7.76 & 1.367 & 1.4795 & 25 & 4 & 67.9 & 55.36 \\
\hline
\end{tabular}

\begin{tabular}{|c|c|c|c|c|c|c|c|c|c|c|c|c|}
\hline \multirow[b]{2}{*}{$\mathrm{T}$} & \multicolumn{6}{|c|}{$\underline{\text { PAN }}$} & \multicolumn{6}{|c|}{$\underline{\text { DMEAF }}$} \\
\hline & $\rho$ & RI & $\mu$ & $\begin{array}{l}\text { DLS Single } \\
\text { particle peak } \\
\text { lifetime (h) }\end{array}$ & $\omega_{o b s}$ & $\frac{\omega_{o b s}}{\omega_{c a l c}}$ & $\rho$ & RI & $\mu$ & $\begin{array}{l}\text { DLS Single } \\
\text { particle peak } \\
\text { lifetime (h) }\end{array}$ & $\omega_{o b s}$ & $\frac{\omega_{o b s}}{\omega_{\text {calc }}}$ \\
\hline 20 & 1.154 & 1.4565 & 89 & $>6$ months & 0.264 & 0.57 & 1.030 & 1.4220 & 8.2 & $>6$ months & 2.86 & 0.49 \\
\hline 25 & 1.151 & & 74 & & & & 1.027 & & 6.9 & & & \\
\hline 30 & 1.148 & 1.455 & 60 & $<1$ & 15.8 & 22.85 & 1.024 & 1.4220 & 5.7 & $<1$ & 79.3 & 9.38 \\
\hline 35 & 1.145 & & 49 & & 12.6 & 14.7 & 1.021 & & 4.7 & & 89.6 & 8.66 \\
\hline 40 & 1.142 & 1.4525 & 39 & & 12.9 & 12.06 & 1.018 & 1.4185 & 3.8 & & 121 & 9.46 \\
\hline 50 & 1.136 & 1.45 & 26 & $<1$ & 5.19 & 3.23 & 1.012 & 1.4150 & 2.5 & $>24$ & 9.96 & 0.51 \\
\hline
\end{tabular}

In our previous manuscript, we presented dynamic light scattering data for EAN using the Malvern ZetaSizer NanoZS, ${ }^{54}$ which showed the persistent presence of a single peak of diameter 1 $\mu \mathrm{m}$ for time periods in excess of 6 months, for dispersions of 1, 2.5, 5, 7.5 and $10 \mathrm{wt} \% 1 \mu \mathrm{m}$ silica spheres in EAN. We concluded from the absence of dimers, trimers, etc. over this time that silica particle suspensions in EAN were stable to aggregation. The data presented in Figure 1 over the much wider accessible size range of the Malvern Instruments Mastersizer 2000 reveals that this was incorrect. Rather, particle flocs with sizes between 50 and $1000 \mu \mathrm{m}$ are present in equilibrium with single particles from the outset. The absence of objects between $1 \mu \mathrm{m}$ and $6 \mu \mathrm{m}$ (here and in our previous DLS measurements) suggests ${ }^{54}$ that flocculation is rapid. The appearance of the particle 
size distribution did not vary appreciably up to 2 hours, at which time the experiment was ended as the water concentration in the sample had risen by $\sim 0.25 \mathrm{wt} \%$. The particle size distributions in all other ILs examined have qualitatively similar behaviour; all systems are comprised of coexisting populations of single particles and flocs. The intensity of single particle scattering peak relative to the floc is lowest for EAN, and in PAN the floc size distribution is narrower.
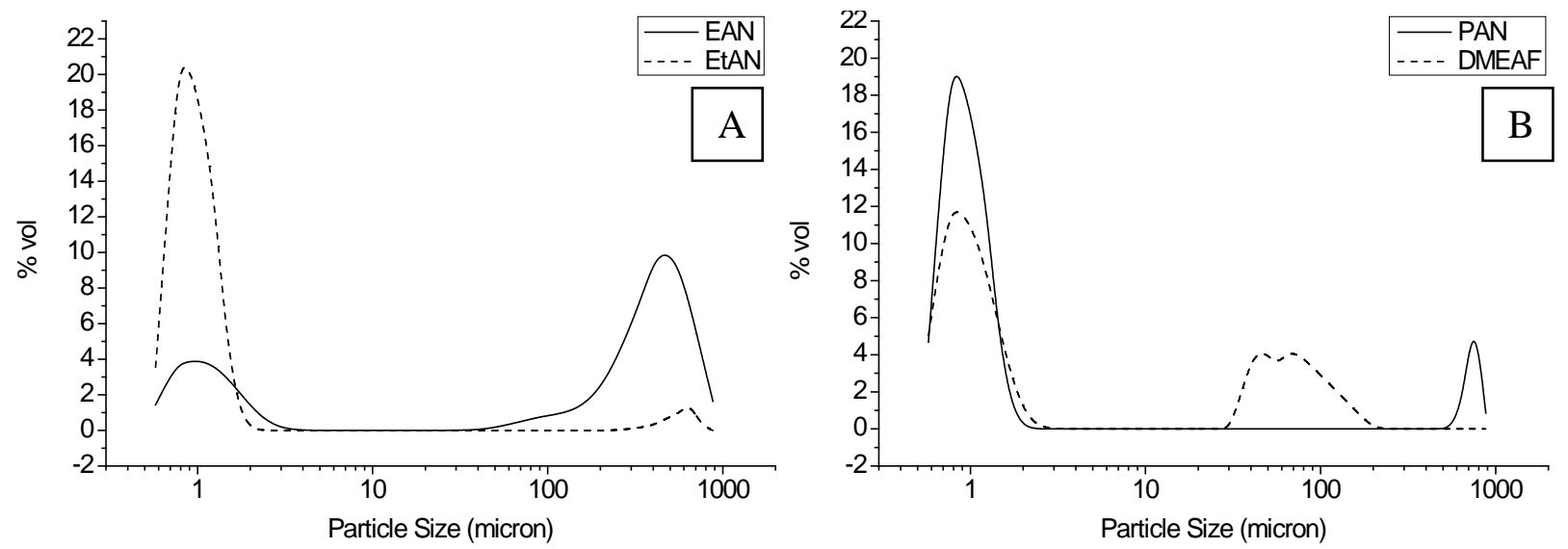

Figure 1 - Static light scattering particle size distributions for $10 \mathrm{wt} \%$ dispersions of $1 \mu \mathrm{m}$ silica particles in (a) EAN and EtAN, and (b) PAN and DMEAF. Measurements recorded at $20{ }^{\circ} \mathrm{C}$, 30 minutes after initial dispersion.

To establish whether singles particles persisted for longer than 2 hours, sealed samples were retained for repeat measurements using the Malvern ZetaSizer NanoZS. Initial experiments were performed at room temperature. After 24 hours, the single particle peak was still present in all of the IL suspensions. These samples were stored at room temperature and measured regularly (after gentle inversion to re-suspend settled particles for light scattering measurements) over a six month time period, and a single particle peak was always detected. The gentle inversion provides minimal mechanical energy to the system and the detected single particles indicate the system is not irreversibly aggregated. These results suggest that single particles are undergoing dynamic exchange with flocculated networks in these ionic liquid suspensions; if aggregation was 
irreversible, eventually all of the particles should join flocs and no peaks corresponding to single particles would remain in the DLS data.

This type of behaviour, in which individual particles are in equilibrium with larger flocs, is characteristic of weak flocculation, which can occur in systems where the attractive and repulsive forces between particles are finely balanced. Because the molten salt nature of the IL effectively reduces the range and magnitude of both the van der Waals and electrostatic forces between particles, ${ }^{49,52}$ the forces associated with IL interfacial structure measured using AFM are extremely small. Colloidal probe measurements between silica surfaces in EAN clearly show that these weak structural forces are the only measurable force present. ${ }^{54}$ Consequently, attractions and repulsions between particles are closely matched, and this means that the energy barrier for a particle to leave a floc is relatively low. We believe this allows weakly flocculated particle suspensions to form in these ILs.

Weakly flocculated systems are rare, and normally occur only over a narrow range of conditions. ${ }^{59}$ Weakly flocculated particle suspensions can be produced in water by using a polymer as a steric barrier to aggregation along with a moderate electrolyte concentration to reduce the particle surface potential. While the van der Waals force is essentially unaffected by the presence of the polymer and electrolyte at this concentration, the repulsive forces are weakened at all particle separations by the presence of salt, and enhanced at short distances due to steric interactions between polymer chains. ${ }^{59}$ The net result is that the attractive and repulsive forces are closely matched, and a weakly flocculated suspension results. The equilibrium kinetics between free particles and aggregates is analogous to evaporation/condensation kinetics at gas-liquid interfaces. ${ }^{59}$

Because attractive and repulsive forces are finely balanced, when thermal motions are increased by raising the temperature, the proportion of single particles increases relative to those in flocs for these aqueous weak flocs. ${ }^{59}$ Thus, the effect of temperature on weakly flocculated IL 
suspensions was investigated using DLS to reveal whether single particles remained in the suspension. When the single particle peak was no longer detected this meant that irreversible aggregation, rather than weak flocculation, had occurred. For EtAN and PAN, increasing temperature markedly reduced the lifetime of single particles in the suspensions, c.f. Table 1. For PAN, when the temperature was increased $30^{\circ} \mathrm{C}$, no single particles could be detected after just 1 hour. The same results were obtained at $50{ }^{\circ} \mathrm{C}$, which was the highest temperature investigated (because nitrous oxide impurities can form in ILs with nitrate anions at higher temperature). ${ }^{55}$ EtAN behaved similarly, except that the transition from weak flocculation to irreversible aggregation occurred at higher temperatures, and that the single particle peak took longer to disappear. As expected, at higher temperatures the flocculated particle suspensions settle much faster than predicted by the Stokes equation for isolated $1 \mu \mathrm{m}$ particles, c.f. Table 1. For these two ILs, the reduction in both range and magnitude of the IL interfacial structural forces on warming, as reported by Wakeham et. al, ${ }^{49}$ which favours aggregation, outweighs the increase in thermal motion that favours single particle dissociation.

The behaviour of silica suspensions in EAN and DMEAF as a function of temperature is more complicated. Increasing the temperature above $\sim 30{ }^{\circ} \mathrm{C}$ again causes the single particle peak to disappear within a few hours, indicating the formation of particle aggregates, and again there is a concomitant increase in the rate of settling compared to Stokes predictions for single particle. However at higher temperatures, single particles are once again stable in the suspension for longer times (at least 24 hours). These results suggest that at low temperature the suspension is weakly flocculated, at intermediate temperatures the ionic liquid interfacial structure is sufficiently reduced such that irreversible aggregates form, but that when the temperature is raised further particle thermal motion is sufficient that the aggregates disocciate more readily and the system returns to a weakly flocculated state. This behaviour is consistent with the repulsive and attractive interfacial forces being finely balanced, so that the establishment of steady-state populations of isolated 
particles and flocs is sensitive to particle diffusion rates. As DMEAF and EAN have lower viscosities, it is possible that at sufficiently high temperatures PAN and EtAN would also reestablish a weakly flocculated state.

The settling behaviour of these IL suspensions in the weakly flocculated state is unusual. When EAN and EtAN are weakly flocculated, they settled at $\sim 6$ times and $\sim 1.5$ times more rapidly than predicted by the Stokes equation for single particles. Settling more rapidly than predicted by Stokes is consistent with enhanced settling of particles when they are part of weakly flocculated aggregates, which suggests that more particles are aggregated in EAN than EtAN. This is consistent with particle size distribution data in Figure 1. Conversely, PAN and DMEAF settle about half as fast as predicted by the Stokes equation when weakly flocculated. To understand this behaviour we have performed oscillatory rheology measurement on each of the suspensions at room temperature; where all of the suspensions are weakly flocculated.

Low shear viscosity data for these ILs without added particles has been reported previously. ${ }^{60}$ The addition of $10 \mathrm{wt} \%$ silica particles did not detectably affect the low shear viscosity of any of these ILs (c.f. supporting information Figure A). For this reason, more sensitive controlled strain oscillatory rheology measurements as a function of frequency were performed, which are presented in Figure 2 for the pure ILs and the ILs with added $10 \mathrm{wt} \%$ silica particles.

At low frequencies for all of the pure ILs, the viscous dissipation or loss modulus, G’, is much greater than the storage or elastic modulus, G'. This indicates energy input to the system is primarily dissipated through movement of the ions as the liquid flows, as expected for the pure solvent. When the silica particles are added to the ILs, there is little change in the appearance of the data, which is consistent with the particles interacting weakly and with flocs exerting little effect on suspension behaviour. As the oscillation frequency increases G' and G” both increase which is typical for a dilute suspension of weakly-interacting spheres. ${ }^{25,61,62}$ At sufficiently high frequencies 
a crossover frequency occurs above which G' exceed G', This indicates a transition from liquidlike to solid-like behaviour where liquid flow alone is unable to dissipate the energy input to the system, and energy is stored elastically by deformation of the liquid or suspension structure. There is minimal movement of the liquid ions or particles on the timescale of the oscillation. Crossovers are observed for DMEAF with and without particles at the same frequency within error, and for EAN in the absence of particles. For all other systems the crossover frequency could not be measured within the accessible frequency range, although it appears the data trend is towards a crossover. Hence all of these systems behave in a liquid-like fashion over a wide frequency range. This is expect for the pure ILs, and consistent with weakly interacting particles in the suspensions. 

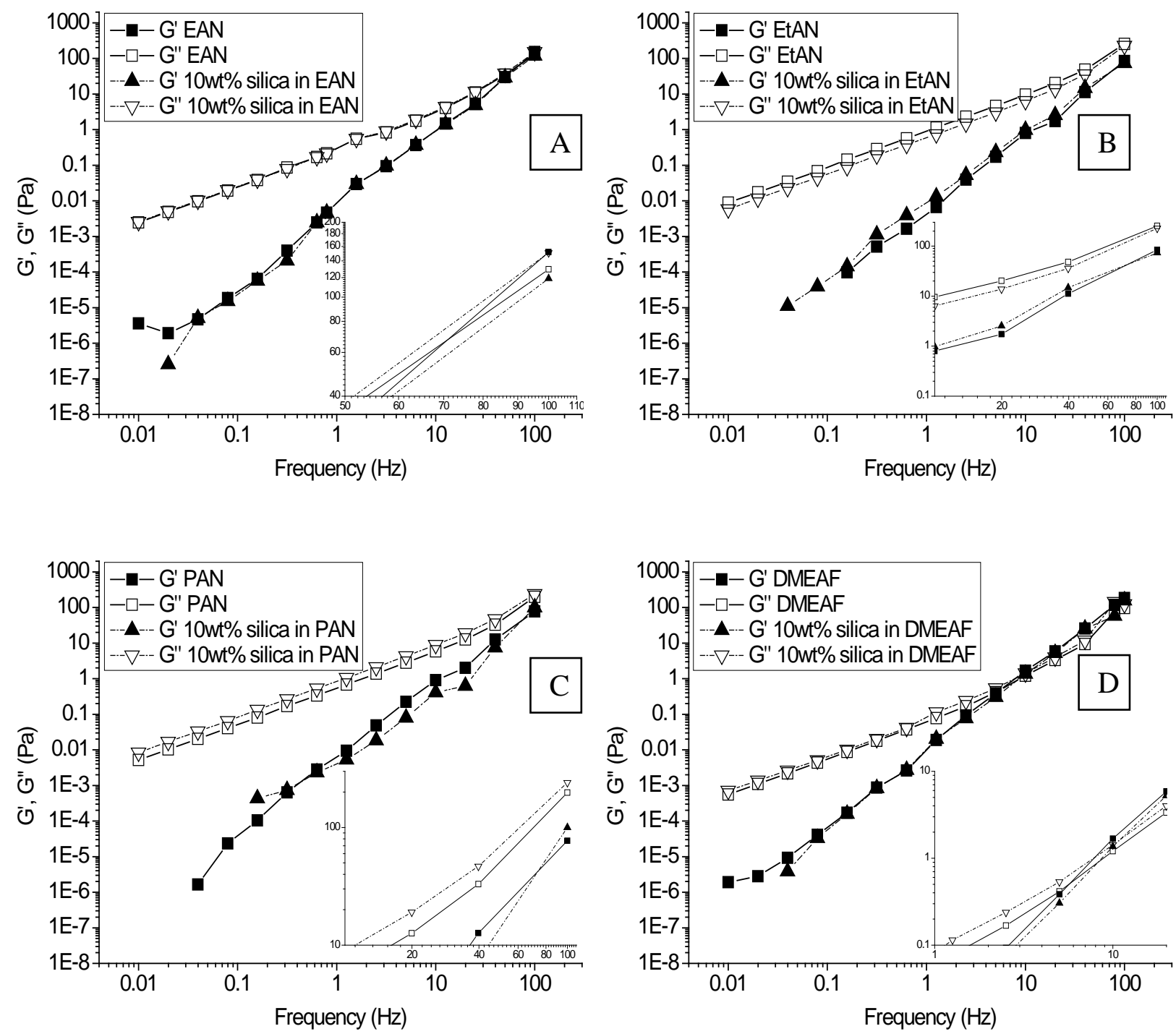

Figure 2 - Controlled strain oscillatory rheology as a function of frequency; pure ILs (square symbols) and dispersions of 10wt\% 1 micron silica (triangular symbols), for EAN (A), EtAN (B), PAN (C), DMEAF (D). Insets are expanded views of the crossover between G' and G” for systems where such occurs and the point of closest approach for systems where it does not.

The phase angle, $\Delta$, is presented as a function of frequency in Figure 3. Delta (equal to the arctangent of G”/G') is a sensitive measure of the elastic character of a material. A delta value of $90^{\circ}$ indicates a purely liquid like response, and delta equal to zero is expected for a solid. Materials with delta values between zero and $90^{\circ}$ are classified as viscoelastic, and have some liquid-like and some solid-like character; the closer delta is to $90^{\circ}$ the more liquid-like the material, and when delta 
is close to $0^{\circ}$ the material is more solid-like. For pure EAN, EtAN and their silica particle suspensions, $\Delta=90^{\circ}$ at low frequencies indicating a purely liquid response. This shows that the weak flocs that form in the suspensions do not affect flow in EAN and EtAN. At higher frequencies $\Delta$ decreases, consistent with the emergence of some elastic or solid-like. For pure DMEAF and PAN at low frequencies, we also find $\Delta=90^{\circ}$ as expected for a pure liquid. However, when $10 \mathrm{wt} \%$ silica particles are added to these ILs, the phase angle decreases, indicating the existence of a persistent structure in the suspension that is able to store energy by deformation even at very low frequencies. This tendency towards a solid-like character is responsible for the slower than Stokes predicted settling in these two systems.
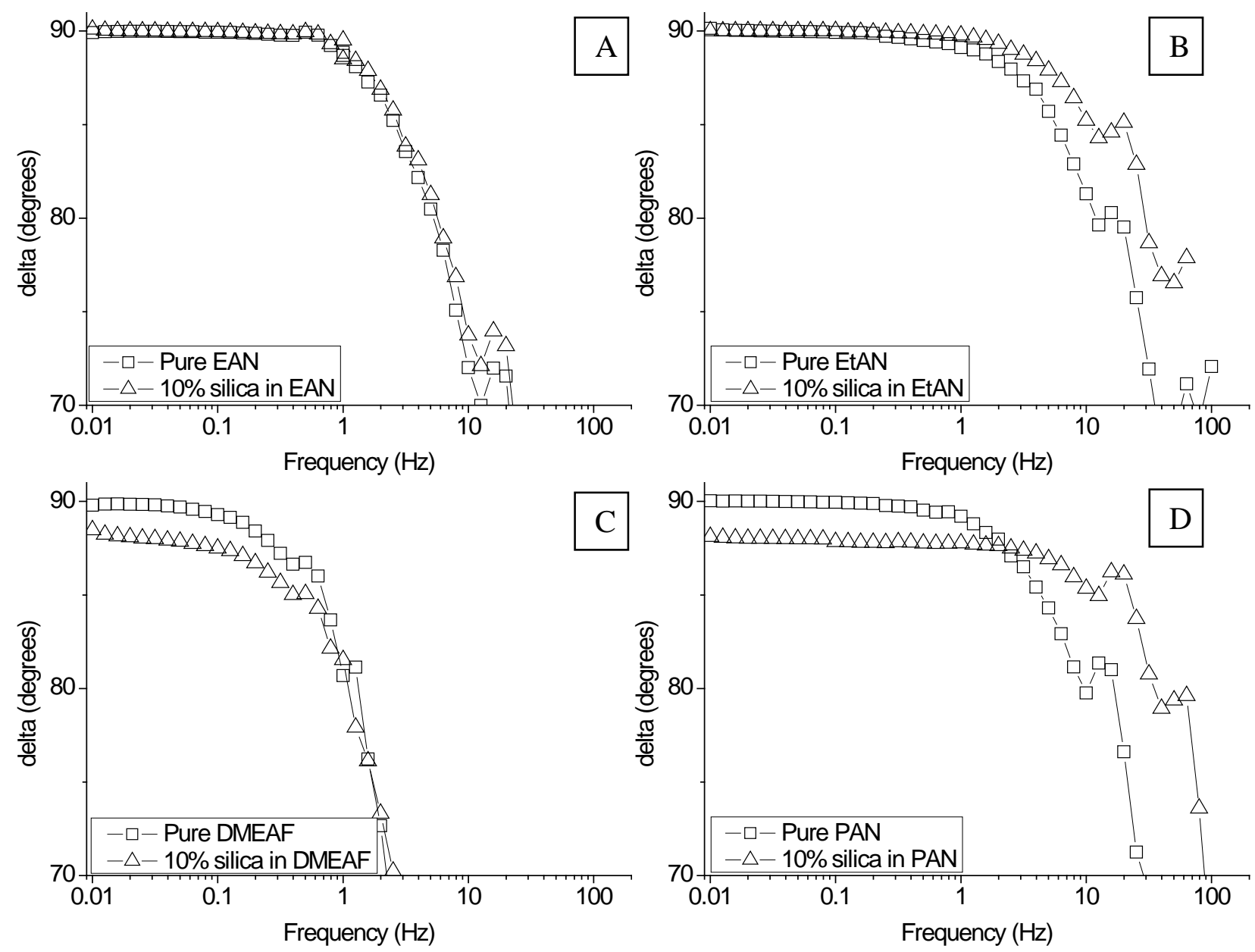

Figure 3 - Delta values corresponding to the viscous and elastic moduli presented in figure 1. For EAN (A), EtAN (B), PAN(C) and DMEAF (D) 


\section{Conclusions}

The repulsive electrostatic and attractive van der Waals forces that operate between particles are both of electromagnetic origin. The pure salt nature of the ILs almost completely screens both of these forces. As such, the only barrier to particle aggregation are weak structural forces derived from near surface IL layers, meaning that contact between particles is expected to be frequent. However, because the van der Waals force is also largely screened, the energy barrier to separation is low, similar to thermal energy. This produces particle suspensions in these ILs that are weakly flocculated; single particles are in equilibrium with flocculated networks, as indicated by the static light scattering data.

Weak flocculation generally exists over a narrow range of conditions because the forces in weakly flocculated systems are finely balanced. For these IL-based weakly flocculated suspensions, when temperature is increased particle thermal motion increases, which favours single particles. However, at the same time structural forces are decreased, which favours particle aggregation. It is the relative rates of change of these two effects with temperature that determines whether the systems remains weakly flocculated or aggregates (with no single particles in suspension). Particle suspensions in all of the ILs are in a weakly flocculated state up to $30{ }^{\circ} \mathrm{C}$. Above this temperature, aggregation occurs, indicated by very rapid settling. Surprisingly, EAN and DMEAF, return to a weakly flocculated state as temperature is further increased the systems because thermal motion breaks particles away from aggregates.

Weakly flocculated systems do not settle according to Stokes law. Suspensions in EAN and EtAN settle more rapidly than Stokes predicts because particles spend an appreciable amount of time in flocs, which settle more rapidly than single particles. Conversely, particles in PAN and DMEAF settle more slowly than Stokes predictions. Oscillatory rheology measurements indicate that $10 \%$ wt silica particle suspensions in PAN and DMEAF have a persistent structure that slows 
settling. Previous AFM studies ${ }^{49,52}$ have revealed that the interfacial structure of PAN and DMEAF is weaker than for EAN and EtAN. Thus the barrier to aggregation is reduced and the persistent structure detected forms.

Weakly flocculated suspensions are unusual. In aqueous systems carefully selected polymers and precise electrolyte concentrations are required to produce the weakly flocculated state. Because ILs so effectively screen the DLVO forces, it is likely many particle suspensions in ILs will exist as weak flocs.

\section{Acknowledgements}

This work was supported by Australian Research Council (ARC) Discovery Projects. RA wishes to acknowledge the ARC for a Future Fellowship. JS wishes to thanks the University of Newcastle for provision of a PhD scholarship.

\section{References}

(1) Welton, T. Room-Temperature Ionic Liquids. Solvents for Synthesis and Catalysis. Chem. Rev. 1999, 99, 2071.

(2) MacFarlane, D. R.; Forsyth, M.; Howlett, P. C.; Pringle, J. M.; Sun, J.; Annat, G.; Neil, W.; Izgorodina, E. I. Ionic liquids in electrochemical devices and processes: managing interfacial electrochemistry. Acc. Chem. Res. 2007, 40, 1165.

(3) MacFarlane, D. R.; Meakin, P.; Sun, J.; Amini, N.; Forsyth, M. Pyrrolidinium Imides: A New Family of Molten Salts and Conductive Plastic Crystal Phases. J. Phys. Chem. B 1999, 103, 4164.

(4) MacFarlane, D. R.; Seddon, K. R. Ionic Liquids-Progress on the Fundamental Issues. Aust. J. Chem. 2007, 60, 3.

(5) Mezger, M.; Schroder, H.; Reichert, H.; Schramm, S.; Okasinski, J. S.; Schoder, S.; Honkimaki, V.; Deutsch, M.; Ocko, B. M.; Ralston, J.; Rohwerder, M.; Stratmann, M.; Dosch, H. Molecular Layering of Fluorinated Ionic Liquids at a Charged Sapphire (0001) Surface. Science 2008, 322, 424.

(6) Rivera-Rubero, S.; Baldelli, S. Surface characterization of 1-butyl-3methylimidazolium Br-, I-, PF6-, BF4-, (CF3SO2)2N-, SCN-, CH3SO3-, CH3SO4-, and (CN)2Nionic liquids by sum frequency generation. J. Phys. Chem. B 2006, 110, 4756.

(7) Triolo, A.; Russina, O.; Bleif, H. J.; DiCola, E. Nanoscale Segregation in Room Temperature Ionic Liquids. J. Phys. Chem. B 2007, 111, 4641.

(8) Triolo, A.; Russina, O.; Fazio, B.; Triolo, R.; Di Cola, E. Morphology of 1-alkyl-3methylimidazolium hexafluorophosphate room temperature ionic liquids. Chem. Phys. Lett. 2008, 457, 362. 
(9) Ueno, K.; Watanabe, M. From Colloidal Stability in Ionic Liquids to Advanced Soft Materials Using Unique Media. Langmuir 2011, 27, 9105.

(10) Asencio, R.; Cranston, E. D.; Atkin, R.; Rutland, M. W. Ionic Liquid Nanotribology: Stiction Suppression and Surface Induced Shear Thinning. Langmuir 2012, 28, 9967.

(11) Angell, C. A.; Byrne, N.; Belieres, J.-P. Parallel developments in aprotic and protic ionic liquids: Physical chemistry and applications. Accounts of Chem. Research 2007, 40, 1228.

(12) Burrell, G. L.; Dunlop, N. F.; Separovic, F. Non-Newtonian viscous shear thinning in ionic liquids. Soft Matter 2010, 6, 2080.

(13) Capelo, S. B.; Mendez-Morales, T.; Carrete, J.; Lopez, L. E.; Vila, J.; Cabeza, O.; Rodriguez, J. R.; Turmine, M.; Varela, L. M. Effect of Temperature and Cationic Chain Length on the Physical Properties of Ammonium Nitrate-Based Protic Ionic Liquids. J. Phys. Chem. B 2012, 116, 11302.

(14) Jacquemin, J.; Anouti, M.; Lemordant, D. Physico-Chemical Properties of NonNewtonian Shear Thickening Diisopropyl-ethylammonium-Based Protic Ionic Liquids and Their Mixtures with Water and Acetonitrile. J. Chem. Eng. Data 2011, 56, 556.

(15) Lopez-Barron, C. R.; Wagner, N. J. Structural Transitions of CTAB Micelles in a Protic Ionic Liquid. Langmuir 2012, 28, 12722.

(16) Lopez-Barron, C. R.; Basavaraj, M. G.; DeRita, L.; Wagner, N. J. Sponge-toLamellar Transition in a Double-Tail Cationic Surfactant/Protic Ionic Liquid System: Structural and Rheological Analysis. J. Phys. Chem. B 2012, 116, 813.

(17) Dupont, J.; de Souza, R. F.; Suarez, P. A. Z. Ionic Liquid (Molten Salt) Phase Organometallic Catalysis. Chem. Rev. 2002, 102, 3667.

(18) Earle, M. J.; Seddon, K. R. Ionic liquids. Green solvents for the future. Pure and Appl. Chem. 2000, 72, 1391.

(19) Ermolaev, V.; Miluykov, V.; Rizvanov, I.; Krivolapov, D.; Zvereva, E.; Katsyuba, S.; Sinyashin, O.; Schmutzler, R. Phosphonium ionic liquids based on bulky phosphines: synthesis, structure and properties. Dalton Trans. 2010, 39, 5564.

(20) Hayashi, S.; Hamaguchi, H.-o. Discovery of a magnetic ionic liquid [bmim]FeCl4. Chem. Lett. 2004, 33, 1590.

(21) Hayashi, S.; Saha, S.; Hamaguchi, H.-o. A new class of magnetic fluids: bmim[FeCl4] and nbmim[FeCl4] ionic liquids. IEEE Trans. Magn. 2006, 42, 12.

(22) Oliveira, F. C. C.; Rossi, L. M.; Jardim, R. F.; Rubim, J. C. Magnetic Fluids Based on I-Fe2O3 and CoFe2O4 Nanoparticles Dispersed in Ionic Liquids. J. Phys. Chem. C 2009, 113, 8566.

(23) Prechtl, M. H. G.; Scariot, M.; Scholten, J. D.; Machado, G.; Teixeira, S. R.; Dupont, J. Nanoscale $\mathrm{Ru}(0)$ Particles: Arene Hydrogenation Catalysts in Imidazolium Ionic Liquids. Inorg. Chem. 2008, 47, 8995.

(24) Triolo, A.; Russina, O.; Bleif, H.-J.; Di Cola, E. Nanoscale Segregation in Room Temperature Ionic Liquids. J. Phys. Chem. B 2007, 111, 4641.

(25) Ueno, K.; Inaba, A.; Kondoh, M.; Watanabe, M. Colloidal Stability of Bare and Polymer-Grafted Silica Nanoparticles in Ionic Liquids. Langmuir 2008, 24, 5253.

(26) Greaves, T. L.; Drummond, C. J. Protic Ionic Liquids: Properties and Applications. Chem. Rev. 2008, 108, 206.

(27) Elbourne, A.; Sweeney, J.; Webber, G. B.; Wanless, E. J.; Warr, G. G.; Rutland, M. W.; Atkin, R. Adsorbed and near-surface structure of ionic liquids determines nanoscale friction. Chem. Commun. 2013, 49, 6797.

(28) Sweeney, J.; Hausen, F.; Hayes, R.; Webber, G. B.; Endres, F.; Rutland, M. W.; Bennewitz, R.; Atkin, R. Control of Nanoscale Friction on Gold in an Ionic Liquid by a PotentialDependent Ionic Lubricant Layer. Phys. Rev. Lett. 2012, 109.

(29) Werzer, O.; Warr, G. G.; Atkin, R. 
Ionic liquid nanotribology: mica-silica interactions in ethylammonium nitrate. J. Phys. Chem. $B$ 2011, 115, 648.

(30) Seddon, K. R. Ionic liquids for clean technology. J. Chem. Technol. Biotechnol. 1997, 68, 351.

(31) Sheldon, R. Catalytic reactions in ionic liquids. Chem. Commun. 2001, 2399.

(32) Wilkes, J. S.; Levisky, J. A.; Wilson, R. A.; Hussey, C. L. Dialkylimidazolium chloroaluminate melts: a new class of room-temperature ionic liquids for electrochemistry, spectroscopy and synthesis. Inorg. Chem. 1982, 21, 1263.

(33) Lehn, J. M. Perspectives in Supramolecular Chemistry-From Molecular Recognition towards Molecular Information Processing and Self-Organization. Angew. Chem., Int. Ed. 1990, 29, 1304.

(34) Crosthwaite, J. M.; Muldoon, M. J.; Dixon, J. K.; Anderson, J. L.; Brennecke, J. F. Phase transition and decomposition temperatures, heat capacities and viscosities of pyridinium ionic liquids. J. Chem. Thermodynamics 2005, 37, 559.

(35) Van Valkenburg, M. E.; Vaughn, R. L.; Williams, M.; Wilkes, J. S. Thermochemistry of ionic liquid heat-transfer fluids. Thermochimica Acta 2005, 425, 181.

(36) Wang, B.; Wang, X.; Lou, W.; Hao, J. Gold-ionic liquid nanofluids with preferably tribological properties and thermal conductivity. Nanoscale Research Lett. 2011, 6.

(37) Jain, N.; Zhang, X.; Hawkett, B. S.; Warr, G. G. Stable and Water-Tolerant Ionic Liquid Ferrofluids. ACS Appl. Materials \& Interfaces 2011, 3, 662.

(38) Pison, L.; Canongia, L. J. N.; Rebelo, L. P. N.; Padua, A. A. H.; Costa, G. M. F. Interactions of fluorinated gases with ionic liquids: solubility of CF4, C2F6, and C3F8 in trihexyltetradecylphosphonium bis(trifluoromethylsulfonyl)amide. J. Phys. Chem. B 2008, 112, 12394.

(39) Earle, M. J.; Esperanca, J. M. S. S.; Gilea, M. A.; Canongia, L. J. N.; Rebelo, L. P. N.; Magee, J. W.; Seddon, K. R.; Widegren, J. A. The distillation and volatility of ionic liquids. Nature 2006, 439, 831.

(40) Rebelo, L. P. N.; Najdanovic-Visak, V.; Gomes, d. A. R.; Esperanca, J. M. S. S.; Nunes, d. P. M.; Guedes, H. J. R.; Visak, Z. P.; de, S. H. C.; Szydlowski, J.; Canongia, L. J. N.; Cordeiro, T. C. Phase behavior and thermodynamic properties of ionic liquids, ionic liquid mixtures, and ionic liquid solutions. ACS Symp. Ser. 2005, 901, 270.

(41) Hayes, R.; Imberti, S.; Warr, G. G.; Atkin, R. Pronounced sponge-like nanostructure in propylammonium nitrate. Phys. Chem. Chem. Phys. 2011, 13, 13544.

(42) Hayes, R.; Imberti, S.; Warr, G. G.; Atkin, R. Amphiphilicity determines nanostructure in protic ionic liquids. Phys. Chem. Chem. Phys. 2011, 13, 3237.

(43) Urahata, S. M.; Ribeiro, M. C. C. Unraveling Dynamical Heterogeneity in the Ionic Liquid 1-Butyl-3-methylimidazolium Chloride. J. Phys. Chem. Lett. 2010, 1, 1738.

(44) Greaves, T. L.; Kennedy, D. F.; Mudie, S. T.; Drummond, C. J. Diversity observed in the nanostructure of protic ionic liquids. J. Phys. Chem. B 2010, 114, 10022.

(45) Greaves, T. L.; Kennedy, D. F.; Weerawardena, A.; Tse, N. M. K.; Kirby, N.; Drummond, C. J. Nanostructured Protic Ionic Liquids Retain Nanoscale Features in Aqueous Solution While Precursor Bronsted Acids and Bases Exhibit Different Behavior. J. Phys. Chem. B 2011, 115, 2055.

(46) Hayes, R.; Imberti, S.; Warr, G. G.; Atkin, R. The Nature of Hydrogen Bonding in Protic Ionic Liquids. Angew. Chem., Int. Ed. 2013, 52, 4623.

(47) Ray, A. Solvophobic Interactions and Micelle Formation in Structure Forming Nonaqueous Solvents. Nature 1971, 231, 313.

(48) Ronis, D.; Martina, E.; Deutch, J. M. The solvophobic effect in simple fluid mixtures. Chem. Phys. Lett. 1977, 46, 53. 
(49) Wakeham, D.; Hayes, R.; Warr, G. G.; Atkin, R. Influence of Temperature and Molecular Structure on Ionic Liquid Solvation Layers. J. Phys. Chem. B 2009, 113, 5961.

(50) Wakeham, D.; Nelson, A.; Warr, G. G.; Atkin, R. Probing the protic ionic liquid surface using X-ray reflectivity. Phys. Chem. Chem. Phys. 2011, 12, 20828

(51) Hamilton, W. A.; Porcar, L.; Butler, P. D.; Warr, G. G. Local membrane ordering of sponge phases at a solid-solution interface. J. Chem. Phys. 2002, 116, 8533.

(52) Atkin, R.; Warr, G. G. Local membrane ordering of sponge phases at a solid-solution interface. Structure in Confined Room-Temperature Ionic Liquids. J. Phys. Chem. C 2007, 111, 5162.

(53) Hayes, R.; Borisenko, N.; Corr, B.; Webber, G. B.; Endres, F.; Atkin, R. Effect of dissolved $\mathrm{LiCl}$ on the ionic liquid-Au(111) electrical double layer structure. Chem. Commun. 2012, $48,10246$.

(54) Smith, J. A.; Werzer, O.; Webber, G. B.; Warr, G. G.; Atkin, R. Surprising particle stability and rapid sedimentation rates in an ionic liquid. J. Phys. Chem. Lett. 2010, 1, 64.

(55) Horn, R. G.; Evans, D. F.; Ninham, B. W. Double-layer and solvation forces measured in a molten salt and its mixtures with water. J. Phys. Chem. 1988, 92, 3531.

(56) Endres, F.; Borisenko, N.; Zein, E. A. S.; Hayes, R.; Atkin, R. The interface ionic liquid(s)/electrode(s): In situ STM and AFM measurements. Faraday Discuss. 2012, 154, 221.

(57) Hayes, R.; El, A. S. Z.; Atkin, R. Pronounced Structure in Confined Aprotic RoomTemperature Ionic Liquids. J. Phys. Chem. B 2009, 113, 7049.

(58) Skinner, B. J.; Appleman, D. E. Melanophlogite, a cubic polymorph of silica. Am. Mineral. 1963, 48, 854.

(59) Long, J. A.; Osmond, D. W. J.; Vincent, B. The equilibrium aspects of weak flocculation, J. Colloid and Interface Sci. 1973, 42, 545.

(60) Smith, J.; Webber, G.; Warr, G. G.; Atkin, R. Rheology of Protic Ionic Liquids and Their Mixtures. J. Phys. Chem. B 2013, 117, 13930,A.

(61) Gravsholt, S. In Polymer Colloids II; Fitch, R., Ed.; Springer US: 1980, p 405.

(62) Barnes, H. A. In An introduction to rheology; Elsevier science pub. Co., 1989. 
TOC Graphic

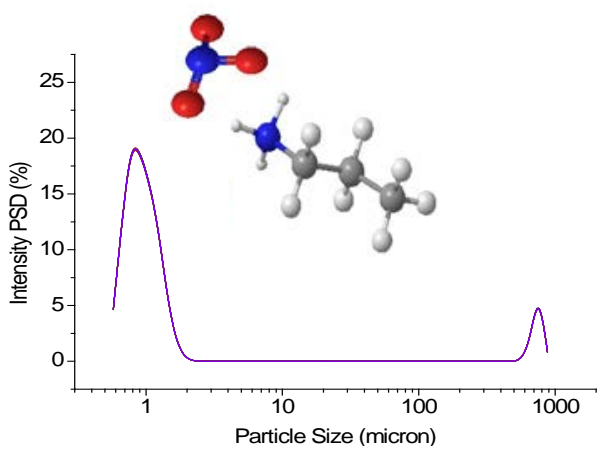

TOC figure - Particle size distribution for 1 micron silica spheres in propylammonium nitrate at equilibrium (at $20^{\circ} \mathrm{C}$ ). 